\title{
Death receptor ligation triggers membrane scrambling between Golgi and mitochondria
}

\author{
S Ouasti ${ }^{1}$, P Matarrese ${ }^{2}$, R Paddon ${ }^{1}$, R Khosravi-Far ${ }^{3}$, M Sorice ${ }^{4}$, A Tinari ${ }^{5}$, W Malorni ${ }^{2,6}$ and M Degli Esposti ${ }^{\star 1,6}$
}

Subcellular organelles such as mitochondria, endoplasmic reticulum (ER) and the Golgi complex are involved in the progression of the cell death programme. We report here that soon after ligation of Fas (CD95/Ap01) in type II cells, elements of the Golgi complex intermix with mitochondria. This mixing follows centrifugal dispersal of secretory membranes and reflects a global alteration of membrane traffic. Activation of apical caspases is instrumental for promoting the dispersal of secretory organelles, since caspase inhibition blocks the outward movement of Golgi-related endomembranes and reduces their mixing with mitochondria. Caspase inhibition also blocks the FasL-induced secretion of intracellular proteases from lysosomal compartments, outlining a novel aspect of death receptor signalling via apical caspases. Thus, our work unveils that Fas ligand-mediated apoptosis induces scrambling of mitochondrial and secretory organelles via a global alteration of membrane traffic that is modulated by apical caspases.

Cell Death and Differentiation (2007) 14, 453-461. doi:10.1038/sj.cdd.4402043; published online 29 September 2006

In many cells, death receptors of the TNF super family, notably CD95/Fas- and DR4/5-binding tumour necrosis factor-related apoptosis-inducing ligand (TRAIL), physiologically mediate cell death via activation of caspases. ${ }^{1,2}$ Death receptors sequentially recruit adaptor proteins such as FADD and apical caspases, in particular procaspase-8, assembling together the death inducing signalling complex (DISC) that promotes activation of apical caspases. In a subset of cells, called type I, ${ }^{1}$ activation of apical caspases is rapid and efficient, involving internalization of active DISC. ${ }^{3,4}$ In all other (type II) cells, mitochondrial factors are required to trigger and amplify the apoptosome-mediated cascade of cell degradation. ${ }^{1,2}$ Mitochondria may also integrate death receptor signalling with other forms of cell death that do not require caspase activation but involve vacuole proliferation, ${ }^{5}$ notably autophagy. ${ }^{6}$ However, it remains unclear how death receptor signalling and autophagy are entwined. . $^{2,6}$

Organelles other than mitochondria are often involved in the progression of cell death induced by diverse stimuli. ${ }^{5,7,8}$ The Golgi complex, for example, has been shown to undergo apoptotic changes in the form of fragmentation or disassembly. ${ }^{8-11}$ Increasing evidence indicates that secretory organelles may be involved also in death receptor-induced apoptosis. ${ }^{8,11}$ Cleavage of structural Golgi proteins (cumulatively termed golgins) is considered responsible for Golgi disassembly during cell death. ${ }^{8}$ However, this is a relatively late event with respect to the involvement of mitochondria, since golgins are predominantly cleaved by effector cas- pases, ${ }^{8-11}$ the activation of which, in type II cells or after intrinsic stimuli of apoptosis, requires mitochondrial factors. ${ }^{2}$ To date, no study has been reported on the relationship between mitochondrial and Golgi organelles during cell death.

A recent paper has indicated that ceramide-derived lipids may be involved in Golgi fragmentation prior to, or parallel to caspase activation. ${ }^{10}$ Ceramide-based glycolipids originating within the Golgi complex have been previously shown to move to mitochondria during death receptor-induced apoptosis. ${ }^{12}$ These and other lipid leads have suggested a hypothetical scenario, in which Golgi and mitochondria interact by local membrane contacts during cell death. ${ }^{5}$ In support of this hypothesis, we report here that FasL induces a global alteration of membrane traffic leading mitochondria to intermix with Golgi membranes in type II cells. We discuss how our results may impinge on diverse pathways of cell death.

\section{Results}

Early Fas activation in type II cells to study organelle mixing. In this work, we applied selected conditions to alter intracellular organelles physiologically, without genetic or pharmacological manipulation as previously used. $4,10,11,13$ Considering that signalling from the DISC is initially restricted to the endocytic membranes carrying internalized receptors and their DISC, ${ }^{3,4,14}$ we have focused our studies on a regime of Fas stimulation enabling the initial activation of

\footnotetext{
${ }^{1}$ Faculty of Life Sciences, The University of Manchester, Stopford Building, Oxford Road, Manchester, UK; ${ }^{2}$ Department of Drug Research and Evaluation, Section of Cell Aging and Degeneration, Rome, Italy; ${ }^{3}$ Department of Pathology, Harvard Medical School, Beth Israel Deaconess Medical Center, Boston, MA, USA; ${ }^{4}$ Department of Experimental Medicine and Pathology, Universita' 'La Sapienza', Rome, Italy and ${ }^{5}$ Technology and Health, Istituto Superiore Sanita', Rome, Italy ${ }^{*}$ Corresponding author: M Degli Esposti, Faculty of Life Sciences, The University of Manchester, Stopford Building, Oxford Road, Manchester M13 9PT, UK. E-mail: mauro.esposti@manchester.ac.uk

${ }^{6}$ Equally senior authors.

Keywords: cell death; membrane traffic; Golgi; mitochondria; secretion

Abbreviations: E64, trans-epoxysuccinyl-L-leucylamido-(4-guanidino)butane; HPA, Helix pomatia agglutinin; IETD, benzyloxycarbonyl-Ile-Glu-Thr-Asp-fluoromethylketone; IVM, intensified video microscopy; MTR, Mitotracker ${ }^{\circledR}$; RB, modified Ringer buffer; TEM, transmission electron microscopy; z-VAD, benzyloxycarbonylVal-Ala-Asp-fluoromethylketone

Received 10.4.06; revised 09.8.06; accepted 10.8.06; Edited by G Salvesen; published online 29.9.06
} 
apical caspases before they target soluble proteins like Bid. ${ }^{15,16}$ Lymphoma type II cells like CEM and Jurkat physiologically very sensitive to Fas-mediated death displayed this situation within $1 \mathrm{~h}$ of FasL treatment, when a 10 -fold increase in caspase-8 activity (Figure 1a) was accompanied by negligible cleavage of its cytosolic

a FasL activation of caspase-8
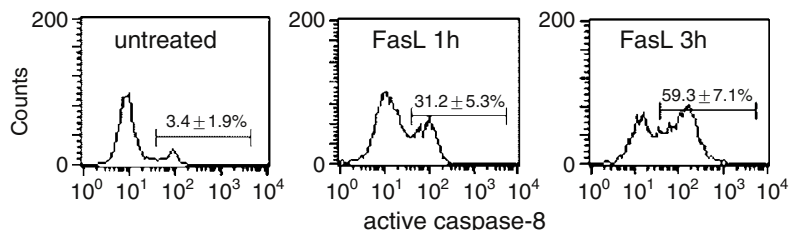

b Golgi vs. mitochondria labelling

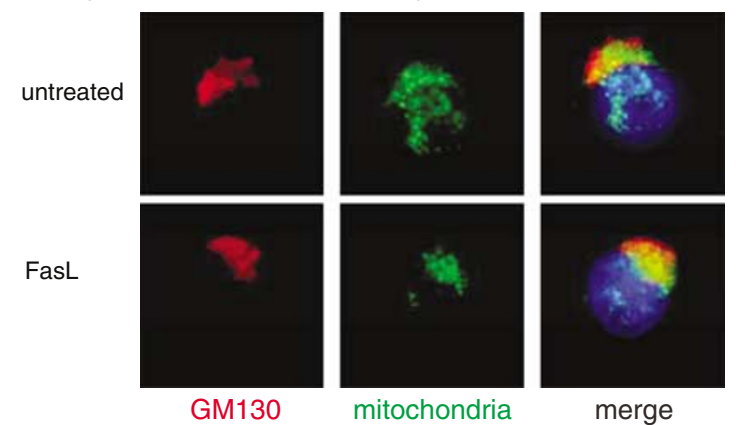

C TEM analysis

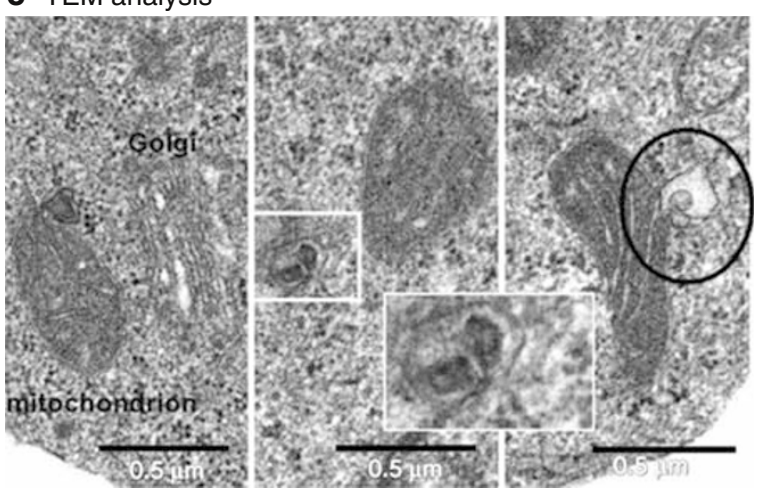

Figure 1 Scrambling of Golgi and mitochondrial membranes after FasL treatment. (a) Activity of caspase-8 was measured in CEM cells by flow cytometry by using the CaspGLOW fluorescein active caspase staining (see Materials and Methods). The assay utilizes the specific caspase inhibitor IETD-FMK conjugated to FITC to label active caspase- 8 within intact cells. The numbers into each panel refer to the percentage of cells displaying enhanced fluorescence attributed to active caspase-8. No significant increase in the basal level of apoptosis ( $<2 \%$ of cells) was detected by dual parametric analysis up to $1 \mathrm{~h}$ of treatment with FasL. (b) Three-colour immunofluorescence images of CEM cells untreated or treated with FasL ( $1 \mathrm{~h}$ as in a) were acquired with IVM technology, that is captured with a CCD camera and a $\times 100$ objective, then processed for background using image analysis software (OPTILAB, Graftek, France). ${ }^{37}$ Nuclei were stained with the Hoechst dye, while Golgi staining was obtained with anti-GM130 monoclonal antibodies (red) and combined with mitochondrial staining (MTR Green, MTR). Note the yellow staining, indicating Golgi/mitochondria overlap, and the partial polarization of mitochondria in treated cells (second row). (c) TEM analysis of the same treated cells as in (b) revealed vesicular events promoted by FasL. Note the close contact between Golgi stalks and a mitochondrion (left panel), directional vesicle budding towards a mitochondrion (middle panel), and a small vesicle merging with a mitochondrion (right panel). Inset: high magnification $(\times 40000)$ of the boxed area in the middle panel substrates, caspase-3 and Bid $\left(\mathrm{cf}^{16}\right)$. Under these conditions, Fas-mediated apoptosis induced localized intermixing of mitochondria and other organelles, evident at both microscopic and subcellular levels.

Intermixing of secretory organelles with mitochondria. We investigated the mutual relationship between mitochondria and Golgi membranes by integrating microscopic, morphological and biochemical approaches (Figures 1 and 2). Images of fluorescence microscopy indicated that, within $1 \mathrm{~h}$ of FasL treatment of $\mathrm{T}$ lymphoma cells, mitochondrial labelling appeared to locally overlap the immunostaining of Golgi-specific membrane markers like GM130 (Figure 1b). Importantly, this occurred before any significant sign of apoptosis (annexin $\mathrm{V}$ positivity) and evident activation of caspase-3 (not shown, cf. $^{16}$ ).

Consistent with fluorescence microscopy results, transmission electron microscopy (TEM) analysis of FasL-treated cells showed an increased frequency of Golgi elements adjacent to mitochondria (Figure 1c, left panel), directional vesicle budding towards mitochondria (Figure 1c, middle) and vacuoles apparently fusing with the outer mitochondrial membrane (Figure 1c, right). To confirm these microscopic results quantitatively, we subsequently evaluated how FasL altered the distribution of non-mitochondrial membrane proteins associated with isolated mitochondria (cf. ${ }^{5,15}$ ). FasL treatment progressively enhanced the mitochondrial levels of endosomal proteins like Rab5 and of Golgi-related proteins like ERGIC53, a membrane marker of the intermediate compartment linking the Golgi with endoplasmic reticulum (ER) (ERGIC ${ }^{17}$ ) (Figure 2ai). When considering the upper band of its immunoblots, ERGIC53 increased also in light membranes (fraction P20) after Fas activation, suggesting a wide dispersal of the protein (Figure 2aii, right). This redistribution occurred concomitantly with an increased mitochondrial association of GM130, the standard marker of Golgi membranes ${ }^{18}$ (Figure 2ii, cf. Figure 1b), which moved from light membranes normally retained within the cytosol-rich fraction.

Next, we refined our cytofluorescence analysis using deconvolution microscopy, which was particularly appropriate for round-shaped T lymphoma cells (Figures 2-4). Contrary to HeLa and other adherent cells displaying heterogeneous Golgi staining, ${ }^{9,11,18}$ over $90 \%$ of untreated Jurkat cells showed a clustered staining of Golgi membranes. However, after 30-40 min of FasL treatment, approx. one-third of cells showed dispersed ERGIC53 staining that partially overlapped with mitochondria, especially towards the cell periphery (Figure 2b). Results obtained with GM130 immunofluorescence further verified the increased contact of secretory and mitochondrial organelles (Figure 2c). Although clustered more tightly than ERGIC53 staining, GM130 staining similarly showed an increased dispersal within $1 \mathrm{~h}$ of FasL treatment (Figure 2c). In cells exhibiting Golgi dispersal, co-staining with mitochondrial markers showed discrete merging of GM130positive elements with mitochondria, especially at the cell periphery (right panels in Figure 2c). Data from deconvolution microscopy were thus consistent with biochemical evidence of increased levels of GM130 in mitochondria-rich fractions of FasL-treated cells (Figure 2a) and also with TEM results (cf. 
a Intermix of Golgi-derived membranes and mitochondria

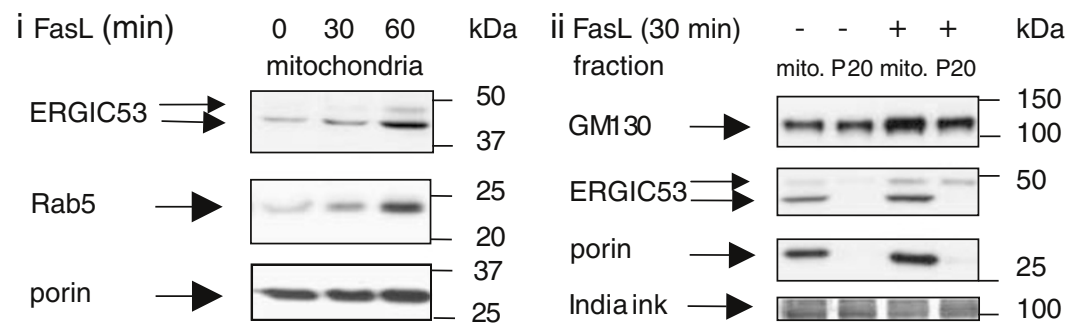

b Deconvolution microscopy
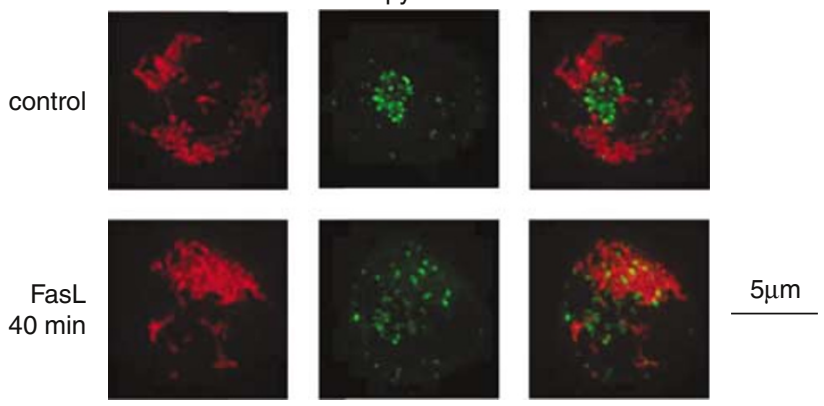

MTR

ERGIC53

Merge
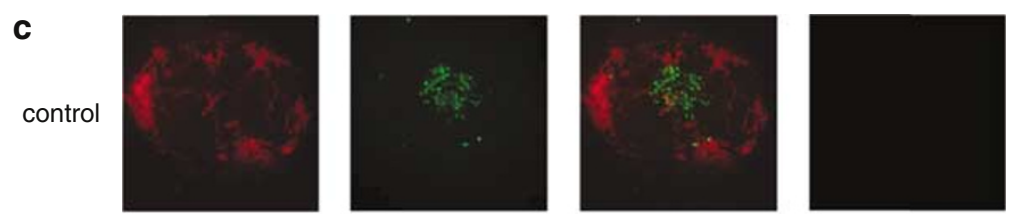

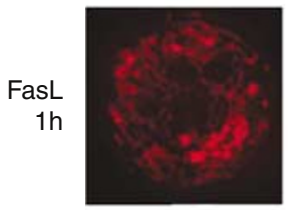

MTR

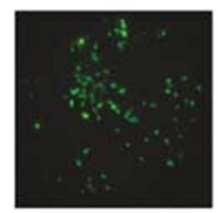

GM130

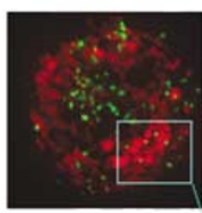

Merge

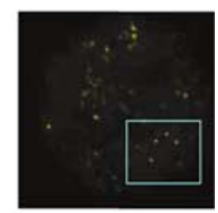

Colocalised

Golgi elements onto mitochondria

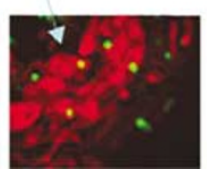

Figure 2 Golgi membranes partially overlap with mitochondria after FasL treatment. (a) Jurkat cells were treated for $30 \mathrm{~min}$ (i) and then $60 \mathrm{~min}$ (ii) with FasL and labelled with diverse membrane markers after isotonic fractionation (cf. ${ }^{15}$ ). Global protein staining with India ink ensued equal loading (bottom panel in ii, right). (b) Projected images from 33 z-sections of $0.2 \mathrm{~nm}$, obtained after 10 cycles of deconvolution (software Rx. 3.4.3, Applied Precision), were acquired by using a state-of-the art Deltavision RT system coupled to an automated Olympus IX71 microscope with a $\times 100$ objective. Cells were first stained with $50 \mathrm{nM}$ MTR red and then processed for ERGIC53 immunostaining. Note the clustering of this staining in untreated cells, reflecting the predominant association of ERGIC elements around the proximal Golgi. ${ }^{17}$ (c) Fluorescence deconvolution images of Jurkat cells before (control) and after FasL treatment for $1 \mathrm{~h}$ were acquired with Deltavision RT as in (b). Mitochondria were first stained with $50 \mathrm{nM}$ MTR and then immunolabelled with a monoclonal anti-GM130 (BD Pharmingen). Projected images from 33 z-sections of $0.2 \mathrm{~nm}$ were obtained with 15 cycles of deconvolution, followed by computer-assisted colour splitting to evidence co-localization using the Deltavision RT software (set with a threshold value of $50 \mathrm{dpi}-$ panels on the right). The insert in the bottom is a magnification of the boxed area in the merged image of dispersed Golgi staining

Figure 1). Of note, GM130 has been associated not only with stacks and cis-Golgi elements, but also with a dynamic compartment probably overlapping with the ERGIC. ${ }^{18}$

FasL globally changes membrane traffic. Our results have been obtained under conditions in which Fas signalling was essentially restricted within endocytic vesicles and their surroundings. ${ }^{4,14}$ Consequently, the observed changes in intracellular organelles (Figures 1 and 2) would be entwined with events of membrane traffic, for instance enhanced pinocytosis. ${ }^{19}$ To evaluate global aspects of endomembrane traffic, we exploited diverse fluorescent derivatives of Helix pomatia agglutinin (HPA), a lectin that recognizes membrane glycoproteins in the ER and proximal Golgi, ${ }^{20}$ as well as at the surface of lymphoma cells. ${ }^{21}$ The latter aspect reflects the traffic of glycoproteins that is modified during apoptosis, in part to stimulate binding of clearing cells. ${ }^{22}$

Static HPA staining of internal membranes partially overlapped with ERGIC53-positive membranes, especially in the 'peri-Golgi' region, and likewise showed dispersal soon after 


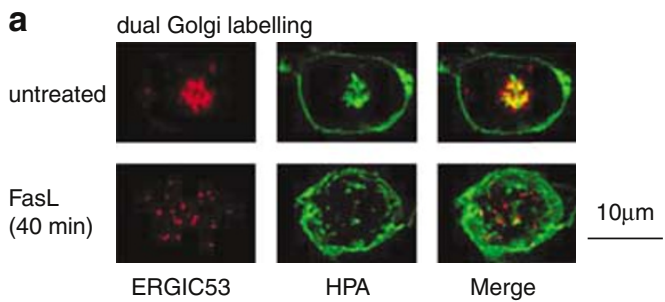

b external HPA vs. GM130

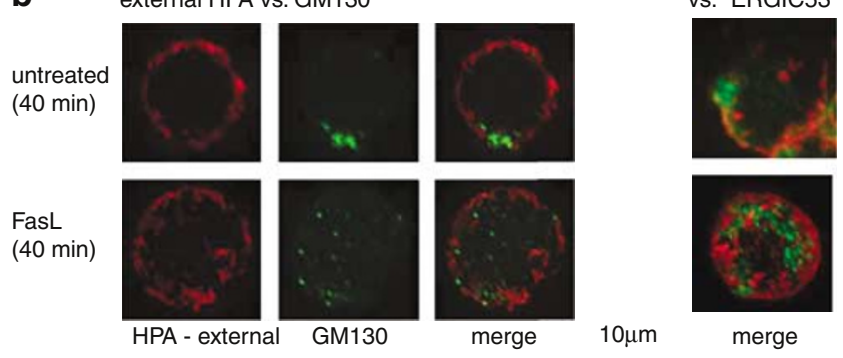

C HPA and mitochondria staining

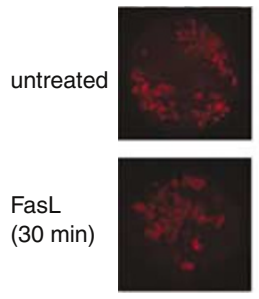

MTR
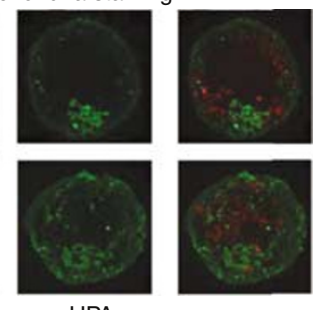

HPA

merge

d HPA binding : mouse liver mitochondria

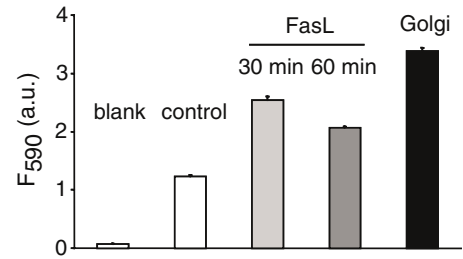

Figure 3 FasL induces global movements of endomembranes. (a) Dual Golgi staining was carried out using Alexa Fluor488-HPA (green) after ERGIC53 immunolabelling that, at difference with the images in Figure $2 b$, was evidenced using a red fluorescent secondary antibody. Projected images from $31 \mathrm{z}$-sections were acquired with a $\times 60$ objective and deconvolved with 10 cycles of the Deltavision RT software. HPA staining was undertaken without lectin preabsorption for a neat definition of the cellular contour. Note the dispersal of this surface staining in the FasL-treated cell. (b) Texas-red-conjugated HPA (HPA external, $20 \mu \mathrm{g} / \mathrm{ml}$ ) was added simultaneously with FasL $(0.5 \mu \mathrm{g} / \mathrm{ml})$ to live cells and incubated for $40 \mathrm{~min}$ in $\mathrm{RB}$ at $37^{\circ} \mathrm{C}$. After washing, cells were fixed, permeabilized and then immunostained with GM130 (left) and ERGIC53 (right - merged images only) as in Figure 2c. Projected images from 34 z-sections were obtained as in (a). (c) Jurkat cells were first loaded with $50 \mathrm{nM}$ MTR (cf. Figure 2), then centrifuged and attached to coverslips, fixed, quenched with unlabelled HPA $(52 \mu \mathrm{g} / \mathrm{ml})$, permeabilized and then stained with $2 \mu \mathrm{g} / \mathrm{ml}$ Alexa Fluor488-HPA (green) to reveal endocellular membranes in preference to surface staining (contrary to a). Projected images from 56 zsections were obtained as in (a). (d) Quantitative measurement of HPA binding to membranes was carried out with mitochondria isolated from mouse liver, treated ex vivo with FasL $\left(\right.$ cf. $^{38}$ ) under the same conditions as those used to induced cell death in lymphoma cells. Texas-red-conjugated HPA $(50 \mu \mathrm{g} / \mathrm{ml})$ was incubated with $40 \mu \mathrm{g} / \mathrm{ml}$ of mitochondrial protein and $8 \mu \mathrm{g} / \mathrm{ml}$ of Golgi protein (black histogram, purified Golgi from rat liver was a kind gift of Dr M Lowe, University of Manchester) for $30 \mathrm{~min}$ in assay buffer at $37^{\circ} \mathrm{C}$. Membranes were then separated and washed by centrifugation, resuspended in assay buffer and transferred in quadruplicate wells for fluorescence measurements in a plate reader (excitation at $544 \mathrm{~nm}$, emission at $590 \mathrm{~nm}$ )
FasL treatment (Figure 3a). However, dispersed HPAlabelled elements diminished their co-localization with scattered ERGIC membranes (Figure 3a), suggesting that Fas stimulation changed the outward traffic of diverse endomembranes of the Golgi complex. Noting that surface staining with HPA increased after FasL treatment, we evaluated the endocytic internalization of external HPA in live cells. External HPA rapidly accumulated underneath the plasmamembrane, without inducing significant cytotoxic effects (Figure $3 \mathrm{~b}$ ). Contrary to the internalization of another agglutinin, ${ }^{23}$ endocytosed HPA hardly labelled the peri-Golgi region of untreated cells (Figure 3). However, FasL treatment greatly increased the intracellular diffusion of internalized HPA, which progressively intermixed with secretory organelles near the cell periphery, as indicated by the increased merging with both GM130- and ERGIC53-labelled membranes (Figure 3b). Hence, internalized glycoproteins of the surface became mixed with elements of secretory organelles early after Fas ligation.

Dispersal of HPA-labelled membranes produced also a diffuse intermixing with mitochondrial staining in FasL-treated cells, increasing close proximity of membranes that were normally well separated (Figure $3 c$ and data not shown). To verify whether this mixing of Golgi-related and mitochondrial membranes was common to cells other than $\mathrm{T}$ lymphomas, we extended our studies to other cells that are physiologically sensitive to FasL, like mouse hepatocytes. ${ }^{15}$ As mouse liver allows the separation of purified organelles in large quantities, we could apply static HPA binding to mitochondria isolated from liver treated with FasL under the same conditions as those used in the previous cellular studies (Figure 3d). Clearly, there was an increase in HPA-binding proteins associated with the membranes of mouse liver mitochondria, which was maximal within $1 \mathrm{~h}$ of FasL treatment (Figure $3 \mathrm{~d}$ ). HPA binding to purified Golgi membranes (black histogram in Figure 3d) provided the positive control for these biochemical measurements that, together with the cellular data above, indicated that HPA-binding elements intermixed with mitochondria during the early phase of Fas signalling. Thus, results obtained in different cell systems strongly supported the concept that Fas activation alters membrane traffic through the Golgi complex, possibly following the opening of a new portal of endocytosis propagating from the cell surface. $^{19,24}$ To investigate this possibility in detail, we expanded our studies on membrane traffic.

Dual HPA labelling of cellular membranes. To test whether FasL treatment deranged membrane traffic along exocytic routes, which can compensate for large changes in membrane surface like those associated with pinocytosis, ${ }^{19,25}$ we combined the internalization of external HPA (Figure 3b) with HPA staining of internal, Golgi-related membranes (Figure 3a,c). The resulting dual HPA staining (Figure 4) enabled the visualization of the global traffic of the same membrane glycoproteins that are involved in surface recognition events. ${ }^{22}$

After FasL treatment, internalized HPA-labelled elements progressively merged with intracellular membranes dispersing away from the peri-Golgi region, indicating accessibility to HPA-binding sites that were normally sequestered (Figure 
a
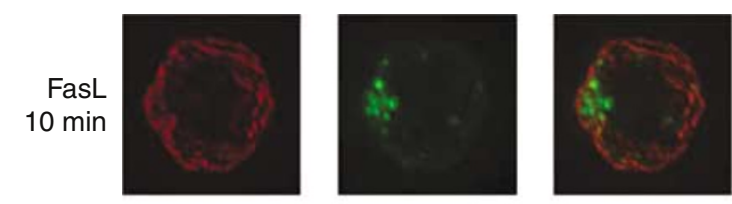

FasL $30 \mathrm{~min}$
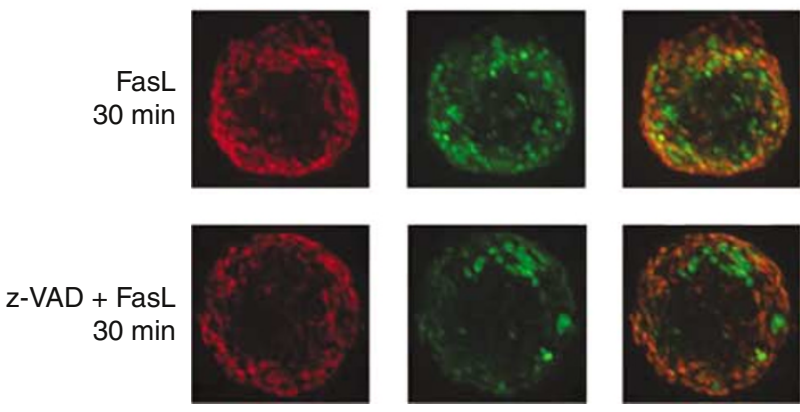

HPA, external

b

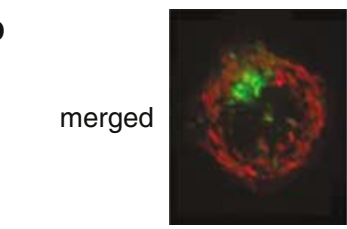

treatment:

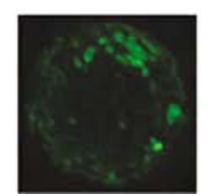

HPA, static

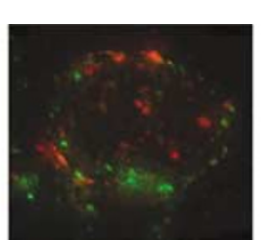

FasL 45 min
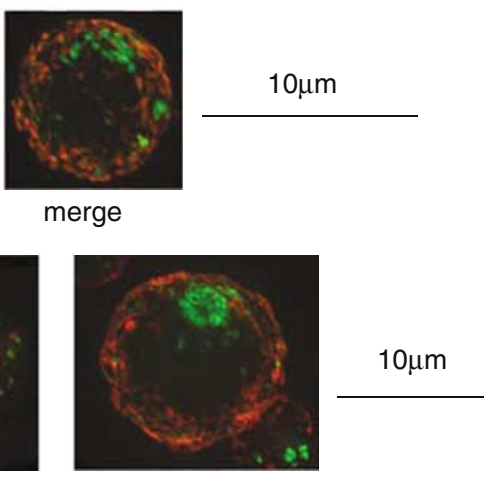

$z-V A D+$ FasL 45 min

C

time-course of surface HPA staining

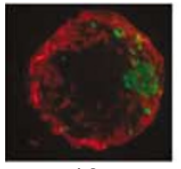

10

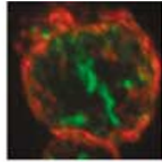

30

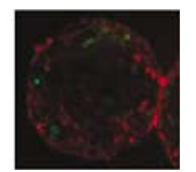

60

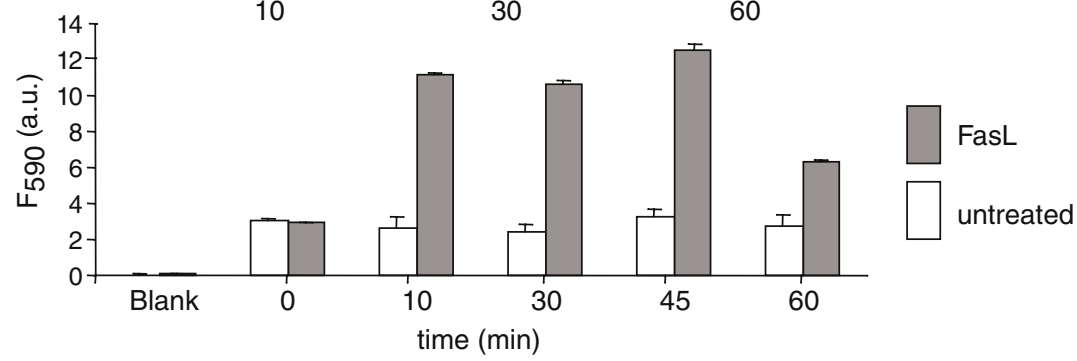

Figure 4 Alteration of membrane traffic and effect of caspase inhibition. (a) Dual staining of mobile membrane elements was accomplished by incubating live cells with Texas-red-conjugated HPA (external, $20 \mu \mathrm{g} / \mathrm{ml}$ ) simultaneously to FasL $(0.5 \mu \mathrm{g} / \mathrm{ml}$ ) as in Figure $3 b$. Aliquots of the same cells were incubated with $50 \mu \mathrm{M} z-\mathrm{VAD}$ prior to FasL addition. Subsequent to fixation, cells were quenched with unlabelled HPA $(52 \mu \mathrm{g} / \mathrm{ml})$, permeabilized and then stained with Alexa Fluor 488-HPA (static HPA, $2 \mu \mathrm{g} / \mathrm{ml}$ for $5 \mathrm{~min}$ ). After washing and mounting, images were obtained with Deltavision RT deconvolution microscopy as in Figure 3c (from 40 to 60 z-sections). (b) Merged images were obtained at later time points in the same experiment as in (a) and derived from deconvolved projections of 34 z-sections. (c) Time-course of HPA uptake and staining of Jurkat cells in the absence and presence of FasL (as in a). Cells were incubated at $37^{\circ} \mathrm{C}$ in RB containing $10 \mu \mathrm{g} / \mathrm{ml}$ of Texas-red-conjugated HPA; at the indicated times, cells were centrifuged in the cold and resuspended in the same buffer without HPA. After counting, 40000 cells of each sample were loaded in quadruplicate wells and measured for the retained fluorescence in a plate reader as in Figure $3 \mathrm{~d}$. Representative images of dual HPA staining as in (a) are inserted for the indicated times of incubation. Note the transient increase of HPA uptake within $1 \mathrm{~h}$

4a,b). Merged staining was maximal approximately $30 \mathrm{~min}$ after FasL addition (Figure 4). Subsequently, internal HPAlabelled membranes also dispersed outside the cells, or reduced their capacity of binding HPA (Figure 4b,c). With regard to the latter possibility, proteolysis of major surface glycoproteins like $C D 43^{22}$ would be more relevant than enhanced de-siaylation of glycoproteins, ${ }^{26}$ given the rapidity of the transient changes (Figure 4c) and the limited $O$ glycosylation capacity of Jurkat cells. ${ }^{27}$ In any case, results with dual HPA staining indicated that FasL treatment induced an early efflux of glycoproteins bound to endomembranes, which transiently intermixed with surface glycoproteins concomitantly entering the cell by enhanced endocytosis.

Caspase modulation of endomembrane movements. If the observed alteration of intracellular membranes and organelles (Figures 1-4) were expression of apoptotic signalling, and not a side effect of Fas activation, it should be influenced by inhibition of caspases, the main executioners of Fas-mediated death. ${ }^{1}$ We thus investigated 
whether inhibition of activated caspases affected the early changes in membrane traffic induced by FasL treatment. Indeed, the pan-caspase inhibitor benzyloxycarbonylVal-Ala-Asp-fluoromethylketone (z-VAD) clearly affected dual HPA staining (Figure 4) under the conditions leading to complete inhibition of caspase-8 activity. ${ }^{16}$ Without influencing the internalization of external HPA, pretreatment with $\mathrm{Z}-\mathrm{VAD}$ reduced the subsequent mixing with internal HPA-positive elements and their outward movements (Figure $4 a, b)$, and also attenuated the Fas-mediated dispersal of GM130-labelled membranes (Figure 5a). Consequently, zVAD inhibited reactions driving the outward movement of secretory endomembranes, similarly to its effect on the transient exposure of glycoproteins involved in binding to macrophages. $^{22}$

Next, we investigated how the outward movement and surface exposure of secretory membranes contributed to the Fas-induced intermixing of Golgi and mitochondrial organelles that we had found earlier (Figures 1-3). To refine our biochemical approach, we isolated extensively purified mitochondria from the nuclear pellets of isotonic homogenates (perinuclear mitochondria, see Materials and Methods). Compared with mitochondria obtained with other methods,

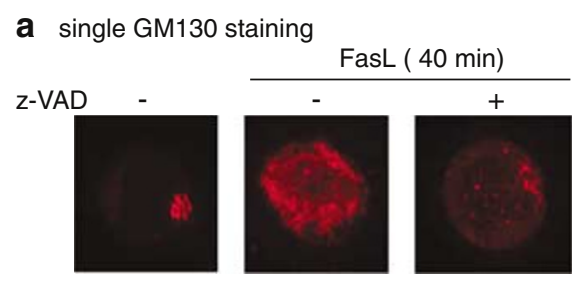

b mixing with perinuclear mitochondria

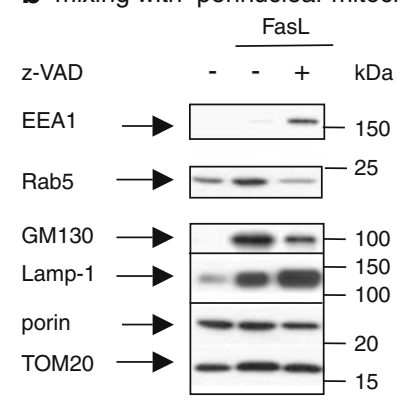

C merged staining with Lamp-1

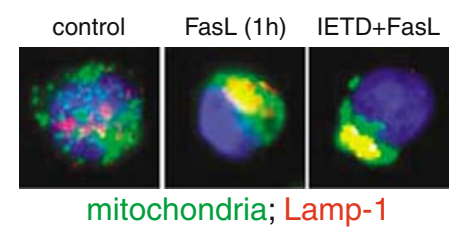

Figure 5 Caspase modulation of subcellular changes in mitochondria and other organelles. (a) Single staining of GM130 was undertaken after 40 min of FasL treatment as in Figure 2c, also after preincubation with $50 \mu \mathrm{M} \mathrm{z-VAD}$, but using red fluorescent secondary antibodies as in Figure 1b. Projected images from $35 \mathrm{z}$ sections were obtained as in Figure $3 b$. (b) Distribution of organelle markers in perinuclear mitochondria from cells treated with FasL for $1 \mathrm{~h}$, also after preincubation with $50 \mu \mathrm{M} z-V A D$ for $30 \mathrm{~min}$. The bottom panels show reblots with marker proteins of the outer mitochondrial membrane. (c) Three-colour immunofluorescence images of cells equally treated with FasL $(1 \mathrm{~h})$ in the absence and presence of $50 \mu \mathrm{M}$ IETD-CHO. IVM data were acquired as in Figure $1 \mathrm{~b}$ these (predominantly perinuclear) mitochondria showed limited contamination from other organelles in untreated cells (cf. Figures $2 \mathrm{a}$ and $5 \mathrm{~b}$ ). FasL treatment for $1 \mathrm{~h}$ increased the levels of GM130, as well as those of the endosomal proteins Rab-5 and Lamp-1 (Figure 5b). However, the same treatment in the presence of $z-V A D$ attenuated the levels of GM130, while enhancing the association of membrane proteins from both early and late endosomes (Figure 5b). Hence, FasL treatment stimulated successive mixing of diverse organelles with mitochondria, in which caspase inhibition affected the association with secretory membranes.

Caspase inhibition additionally induced a diversion of the membrane traffic converging onto mitochondria, favouring association with endo-lysosomes (Figure 5b and data not shown). It would then appear that blockage of endomembrane efflux (Figures 4 and 5a) produced a congestion of the membrane traffic initially altered by Fas signalling, an interpretation that was confirmed by results obtained at the cellular level. For instance, we observed an increased overlapping of Lamp-1 and mitochondrial staining in immunofluorescence images of cells treated with FasL in the presence of benzyloxycarbonyl-Ile-Glu-Thr-Asp-fluoromethylketone (IETD), an inhibitor most specific for caspase-8 (Figure 5c).

While we could not find any significant cleavage of structural Golgi proteins that correlated with the intermixing of organelles (Figure $5 \mathrm{~b}$ cf. ${ }^{11}$ ), we observed some cleavage of BAP31 associated with mitochondria (results not shown). BAP31 is an abundant protein of ER and Golgi membranes ${ }^{28}$ that facilitates the retention of proteins within early secretory organelles $^{29,30}$ and has been reported to be cleaved by caspase-8. ${ }^{13}$ However, BAP31 cleavage was limited and did not extend to all membrane fractions, thereby reducing the possibility of its direct involvement in the traffic alteration observed here.

Release of lysosomal proteases is accelerated by caspases during Fas signalling. As a natural extension of our novel findings of caspase-mediated efflux of secretory membranes (Figures 4 and 5), we evaluated whether FasL treatment affected exocytic events by following the lysosomal granules that are rapidly secreted by focal exocytosis. ${ }^{25}$ In Jurkat cells, these granules were efficiently labelled with BODIPY TR-conjugated casein (EnzChek ${ }^{\circledR}$, Figure 6a), a self-quenched protein functioning as a substrate of secretory proteases. ${ }^{31}$ In untreated cells, secretory granules loaded with EnzChek decreased in number and distribution only slowly (Figure 6a, compare top and bottom panels of red staining). Treatment with FasL significantly reduced the number and distribution of labelled granules (Figure 6a,b). However, pretreatment with z-VAD completely blocked the loss of EnzChek-labelled granules (Figure 6a,b), while partially enhancing the surface staining of HPA (Figure 6a, middle panels; cf. Figure 4b).

To verify whether the disappearance of peripheral lysosomal granules derived from increased secretion by FasLtreated cells, we simultaneously stained cells with EzCheck and Rhodamine110-FR-bisamide, a fluorogenic substrate most specific for cathepsin L. ${ }^{32}$ Contrary to other ubiquitous cathepsins, cathepsin $L$ is only partially directed to lysosomes and undergoes facile secretion, ${ }^{33}$ as indicated in our cells by 
the limited co-localization of its green-fluorescent substrate with peripheral granules stained by EnzChek (Figure 6b). Nevertheless, FasL treatment dramatically altered the subcellular distribution of cathepsin $L$ activity, inducing a diffuse pattern of intracellular staining (Figure $6 b$ ) in part resembling that reported previously in dying T cells. ${ }^{34}$

Moreover, FasL treatment increased the green fluorescence dispersed outside FasL-treated cells, indicating exocytic secretion of cathepsin L. Indeed, the supernatant of FasL-treated cells showed significant levels of protease activity, as determined with either EnzChek (Figure 6c) or the cathepsin L-specific substrate (Figure 6d). Consistent with previous data (Figures 4 and $6 a$ ), pretreatment with z-VAD strongly reduced the overall dispersal of cathepsin $L$ activity (Figure $6 \mathrm{~b}$ ) and the protease activity in cell supernatants (Figure 6c,d). Conversely, the fluorescence of intracellular EnzChek showed a slow decrease after FasL treatment, when continuously monitored in a fluorimeter (Figure 6c, bottom).

Taken together, our data indicated that FasL treatment strongly increased the secretion of lysosomal granules and of a major acid hydrolase, cathepsin L, from cells physiologically sensitive to FasL-mediated cell death. The enhanced exocytic activity was fundamentally mediated by early activation of caspase-8, which was fully inhibited by z-VAD under our conditions. Despite similarities in the subcellular distribution observed by cytofluorescence, our results contrasted with those reported by Michallet et al., ${ }^{34}$ which showed intracellular dispersal of cathepsin $L$ independently of caspase activation. In any case, this is first time that Fas signalling has been
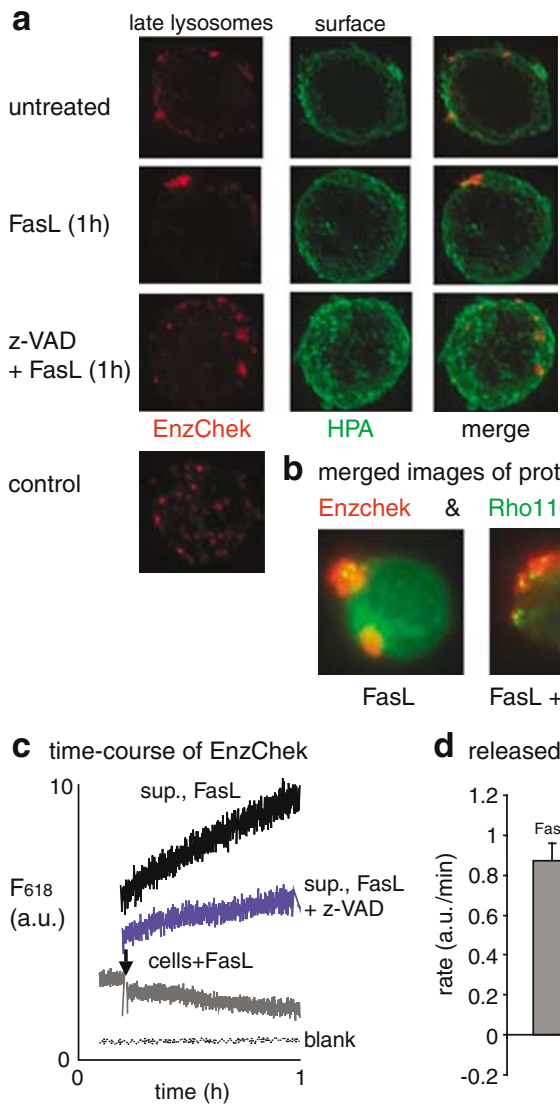

merged images of protease staining Enzchek \& Rho110-FR-bisamide 안.

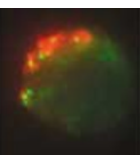

$$
\text { FasL + z-VAD }
$$
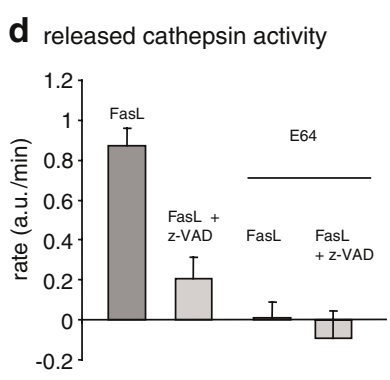

shown to induce an early release of lysosomal proteases outside cells.

\section{Discussion}

By studying the early dynamics of intracellular membranes in Fas-mediated apoptosis, we have discovered a rapid engagement of membrane organelles in type II cells. In particular, FasL treatment induces intermixing of Golgi and mitochondrial organelles (Figure 1). Although indirect evidence for 'scrambling' of Golgi membranes has been reported previously (reviewed $\mathrm{in}^{5,8}$ ), our data provide the first demonstration of Golgi-mitochondria intermixing in cell death. In extending our studies, we have clarified that this scrambling is not an isolated phenomenon, nor is restricted to lymphoid cells. In fact, it was detected in cells growing in suspension cells like CEM as well as adhering cells such as hepatocytes (Figure 3d) and HeLa lines. The intermixing of membrane organelles also preceded any specific alteration of main cytoskeleton components, that is actin and tubulin, either in lymphoma or in Hela adhering cells ( $P$ Matarrese and W Malorni, unpublished data). Hence, the scrambling of diverse organelles occurs early after activation of Fas and appears to

Figure 6 Secretion of lysosomal markers is accelerated by caspases during Fas signalling. (a) Jurkat cells were incubated for $15 \mathrm{~min}$ at $37^{\circ} \mathrm{C}$ in RB containing $10 \mu \mathrm{g} / \mathrm{ml}$ of BODIPY TR-conjugated casein, a fluorogenic substrate of proteases (EnzChek ${ }^{\circledR 31}$ ), then washed and chased in full growth medium for $1 \mathrm{~h}$. After resuspension in RB, in the absence of presence of Z-VAD (incubated for $20 \mathrm{~min}$ ), cells were left untreated or treated with FasL for $1 \mathrm{~h}$ as in the experiment of Figure $5 \mathrm{~b}$. After fixation, cells were briefly stained with $2 \mu \mathrm{g} / \mathrm{ml}$ Alexa Fluor488-HPA (green) to evaluate surface changes. In untreated cells, EnzChek stained an array of peripheral and cortical granules (usually 6-8 per cell) that remained relatively stable after hours of chase and corresponded to secretory lysosomes (the bottom panel shows a representative image of control cells immediately after the chase). Fas-activation strongly decreased the number of these granules, whereas z-VAD inhibition of caspases restored their normal frequency and distribution. Deconvolved images were obtained from 54 z-sections as in Figure 3c. (b) Jurkat cells were double labelled with fluorogenic substrates of proteases. Staining with EzCheck was performed as in (a), but simultaneously with the uptake of $10 \mu \mathrm{M}$ Rhodamine110FR-bisamide, a fluorogenic substrate of cathepsin L. ${ }^{32}$ This substrate produced green fluorescence that was confined in a few small vacuoles, some of which colocalized with those labelled by EnzChek. Treatment with FasL for $1 \mathrm{~h}$ induced diffused green fluorescence within and outside the cells (left panel), in part due to cathepsin release from lysosomal compartments. ${ }^{34}$ However, pretreatment with zVAD strongly reduced this diffusion of green fluorescence while increasing its focal concentration in vacuoles overlapping secretory lysosomes (right panel). Merged images were projections of $52 \mathrm{z}$-sections that had not been deconvolved, to better show the diffused pattern of green fluorescence. (c) Cells were incubated with EnzChek and then chased as in $(\mathbf{a}, \mathbf{b})$; after washing, they were resuspended at $2 \times 10^{6} / \mathrm{ml}$ in RB and the fluorescence of intracellular EnzChek was continuously monitored at $24^{\circ} \mathrm{C}$ in a fluorimeter, subsequently to FasL addition (arrow). After $1 \mathrm{~h}$, the same cells were centrifuged and their supernatant was supplemented with fresh EnzCheck ( $20 \mu \mathrm{g} / \mathrm{ml})$ to measure the activity of released proteases (again at $24^{\circ} \mathrm{C}$ ). Compared to control cells (not shown) or z-VAD-treated cells (middle trace), the supernatant of Fas-treated cells showed a strong increase in protease activity. The dotted line at the bottom represents the blank containing EnzChek alone. (d) Cathepsin $L$ activity was measured in supernatants of cells treated with Fas $L$ for $45 \mathrm{~min}$, in the absence and presence of z-VAD as in (b) (cf. Figure 4) using $1 \mu \mathrm{M}$ Rhodamine110-FR-bisamide at $37^{\circ} \mathrm{C}$ and $\mathrm{pH}$ 6.0. Mean rates $(n=3)$ were measured using a plate reader as in Figure 4c. The histograms on the right show the strong decrease of activity measured in the presence of $5 \mu \mathrm{M} \mathrm{E}-64$, a general inhibitor of cathepsins 
reflect a global alteration of membrane traffic in diverse type II cells, albeit being particularly rapid in cells physiologically sensitive to Fas-mediated death (Figures 1-5).

Of note, the dispersal of secretory organelles that we have presented here differs from the disassembly of the Golgi complex that has been reported previously ${ }^{8-11}$ because it occurs transiently (Figure 4), involving apical caspases (Figures 4-6) but without apparent cleavage of golgins (Figures $2 \mathrm{a}$ and $5 \mathrm{~b}$ ). The early events we have observed, on the other hand, may be related in part to the dispersal of Golgi and ERGIC organelles produced by treatment with exogenous ceramide, ${ }^{10}$ which artificially alters membrane traffic and the lipid composition of diverse organelles. ${ }^{5}$ Although the link between ceramide generation and Fas signalling remains controversial, ${ }^{5,10}$ physiological connections normally link Golgi and mitochondrial membranes in the traffic and distribution of ceramide-based glycolipids, which becomes deranged during death receptor-mediated apoptosis. ${ }^{12}$ Lipid trafficking thus provides an underlying platform for a limited or transient association of Golgi and mitochondrial membranes. ${ }^{5}$ The death receptor-induced increase in membrane traffic, partially driven by endocytic events, ${ }^{3-5,19,24,35}$ may abnormally alter the direction and intensity of inter-organelle contacts, distorting the normal flow of membrane interactions. This would provide a simple explanation for the increased frequency of local intermixing of Golgi and mitochondrial membranes that we have documented here (Figures 1-3).

Conversely, the emerging role of ER perturbation in mitochondria-dependent cell death ${ }^{5,7,10}$ suggests that ER compartments connected with Golgi and mitochondrial membranes may transmit Fas-induced changes in membrane traffic, as in the case of T-cell receptor signalling. ${ }^{35}$ For instance, Fas signalling may impact on secretory routes linking ER with proximal Golgi by early modification of BAP $31^{13}$ or similar traffic regulators that retain selected membrane proteins within the ER. ${ }^{29}$ Functional alteration of these ER retention proteins via caspase cleavage could induce a dispersal and surface exposure of specific membrane proteins, ${ }^{29,30}$ that may include glycoproteins reacting with HPA. ${ }^{36}$

Our finding that inhibition of (predominantly) caspase-8 prevents early dispersal of Golgi membranes provides a novel dimension to the early action of apical caspases, especially in type II cells. Of note, a caspase-dependent release of intracellular proteases (Figure 6) has not been observed before in death receptor-mediated apoptosis. Without attempting speculations on the possible implications for a constitutive activity of caspase-8, we wish to address the inevitable question: what would be the physiological relevance of caspase-mediated disregulation of endomembrane traffic during cell death? One possibility is that it could open an early window of communication with other cells by exposing intracellular membrane lipids ${ }^{15}$ and carbohydrates. ${ }^{22,36}$ Moreover, the stimulation of outward movements may also potentiate death signalling by mobilizing Fas receptors from the internal stores within the Golgi apparatus. ${ }^{8}$ Most importantly, however, caspase stimulation of endomembrane movements could minimize the spontaneous maturation of endosomes into lysosomes that may affect mitochondria via caspase-independent reactions (Figure $5 b a^{2,5}$ ). Hence, early activation of apical caspases effectively reduces the extent to which other forms of cell death may emanate from the DISC.

Following the discussion above, we believe that our work may impinge on caspase-independent cell death as well, since inhibition of apical caspases attenuates exocytic movements (Figures 4 and 5) and blocks the release of lysosomal material (Figure 6). The immediate consequence of caspase inhibition is then an accumulation of intracellular endolysosomes, which could ultimately facilitate self-degradation of cells and trigger autophagy. ${ }^{6}$ To conclude, we present here novel evidence that Fas signalling produces global changes in the membrane traffic of intracellular organelles, which could influence both caspase-mediated and caspase-independent cell death.

\section{Materials and Methods}

Reagents. Fluorescent probes and assays were purchased from Molecular Probes/Invitrogen (Eugene, OR, USA). Commercial antibodies were obtained from Santa Cruz Biotechnologies (Santa Cruz, CA, USA) and BD-Pharmingen (Oxford, UK). The following antibodies were generous gifts of colleagues: $\mathrm{Dr} V$ Lennon (Mayo Clinic, USA, for monoclonals against BAP-31); Dr H Hauri (Basel, Switzerland) for a monoclonal against ERGIC53; Dr M Lowe, A Gilmore, M Poole and P Woodman (Manchester, UK) for Golgi, mitochondrial, ER and endosomal antibodies, respectively. D609 was from Biomol (Plymouth Meeting, PA, USA) and $z$-VAD from Alexis (San Diego, CA, USA). Recombinant superFasL was purchased from Apotech (Lausanne, Switzerland). Other chemicals were from Sigma (St Louis, MO, USA), GibCo and Fisher (Loughborough, UK).

Cell culture and apoptosis stimulation. Human T-lymphoma cells, CEM and Jurkat, were cultured in RPMI medium containing 10\% foetal calf serum (FCS). HeLa cells were cultivated as described earlier. ${ }^{16}$ Cells were treated with recombinant FasL in the absence of serum and the levels of apoptosis were evaluated by bi-parametric flow cytometry analysis ${ }^{17,37}$ using a FACScan instrument (Becton Dickinson, Franklin Lakes, NJ, USA).

Caspase measurements. Activation of caspases was evaluated in live cells by flow cytometry using CaspGLOW fluorescein active caspase staining (MBL, Woburn, MA, USA). The assay utilizes the cell-permeable caspase inhibitors IETDfmk and DEVD-fmk conjugated to FITC as fluorescent markers, which irreversibly bind to the active forms of caspase- 8 and -3 , respectively. Complementary assays were performed in subcellular fractions using fluorogenic substrates of caspases. ${ }^{17,38}$

Organelle isolation. Isotonic subcellular fractionation was carried out essentially as described. ${ }^{20}$ Cells were homogenized in isolation buffer $(0.25 \mathrm{M}$ mannitol, $1 \mathrm{mM}$ EDTA, $10 \mathrm{mM} \mathrm{K-HEPES,} 0.2 \% \mathrm{BSA}$, pH 7.4) containing a cocktail of protease inhibitors (further supplemented with $50 \mu \mathrm{M} z-V A D$ to minimize caspaseinduced degradation of proteins during fractionation). Postnuclear supernatants were centrifuged at $10000 \times g$ for $10 \mathrm{~min}$ and the pellets were kept as fraction P10 (suspended in assay buffer: $0.12 \mathrm{M}$ mannitol, $0.08 \mathrm{M} \mathrm{KCl}, 1 \mathrm{mM}$ EDTA, $20 \mathrm{mM}$ K-HEPES, pH 7.4), or recentrifuged to obtain mitochondria and their wash (fraction S3). The supernatants were centrifuged at $22000 \times g$ for $50 \mathrm{~min}$ to separate light membranes (fraction P20) from the bulk of the cytosol (fraction S20). Extensively purified mitochondria were obtained from the nuclear pellet that was rehomogenized in assay buffer and centrifuged at $600 \times \mathrm{g}$. The supernatant $(0.4 \mathrm{ml})$ was then layered onto a cushion of $1 \mathrm{M}$ mannitol $(0.6 \mathrm{ml}$, covered with $0.1 \mathrm{ml}$ of $2 \% \mathrm{BSA}$ in assay buffer). After centrifugation at $9000 \times g$ for $15 \mathrm{~min}$ at $4^{\circ} \mathrm{C}$, the pellet was resuspended in assay buffer, centrifuged at $10000 \times g$ for $10 \mathrm{~min}$, and then resuspended in $25 \mu \mathrm{l}$ of assay buffer with protease inhibitors. A similar procedure was used to fractionate mouse liver. ${ }^{38}$ After evaluation of protein concentration, ${ }^{38}$ fractions were stored at $-80^{\circ} \mathrm{C}$.

Immunoblotting. Cell lysates and subcellular samples were separated by SDS-polyacrylamide gel electrophoresis and immunoblotted as described previously. ${ }^{15,38}$ 
Organelle staining. Mitochondria and other organelles were specifically stained either in solution (for intensified video microscopy (IVM) analysis ${ }^{37}$ ) or after attachment to polylysine-coated coverslips ${ }^{35}$ with Mitotrackers (MTR green or red) and fluorescent lectins. ${ }^{21}$ After $10-60 \mathrm{~min}$ incubation, cells were fixed with $4 \%$ paraformaldehyde ( $w / v$ in PBS), permeabilized with Triton-X100, blocked, incubated with monoclonal antibodies for $1 \mathrm{~h}$, washed again and then incubated for 45-60 min with anti-mouse secondary antibodies conjugated with Alexa Fluor 488 or Rhodamine $\mathrm{X}$. See figure legends for details of fluorescence microscopy imaging. Staining of late lysosomes was performed with the protease substrates EnzChek ${ }^{\circledR}$ (BODIPY TR-casein; ${ }^{31}$ see the legend of Figure 6).

Fluorescence-based assays. Endocytosis was evaluated following the internalization of fluorescent HPA, which binds to surface glycoconjugates of T. lymphoma cells. ${ }^{21}$ Measurements were carried out in a plate reader with appropriate filters (Fluoroskan Ascent, Thermo, Basingstoke, UK). Endocytosis was also measured following the fluorescence changes of FM1-43 ${ }^{35}$ in a plate reader, using $5 \times 10^{5} \mathrm{cell} / \mathrm{s} / \mathrm{ml}$ in a modified Ringer buffer (RB, containing $145 \mathrm{mM} \mathrm{NaCl}, 4.5 \mathrm{mM}$ $\mathrm{KCl}, 2 \mathrm{mM} \mathrm{MgCl}_{2}, 1 \mathrm{mM} \mathrm{CaCl}_{2}, 5 \mathrm{mM} \mathrm{K}$-HEPES, pH 7.4, and $10 \mathrm{mM}$ glucose) and $2-4 \mu \mathrm{M}$ FM1-43. The activity of cathepsin $\mathrm{L}$ was measured using the substrate rhodamine110-FR-bisamide. ${ }^{32}$ Other cathepsin assays were undertaken as described. ${ }^{34}$

Transmission electron microscopy studies. For TEM examination, cells were fixed in $2.5 \%$ cacodylate-buffered $(0.2 \mathrm{M}, \mathrm{pH} 7.2)$ glutaraldehyde for $20 \mathrm{~min}$ at room temperature and postfixed in $1 \% \mathrm{OsO}_{4}$ in the same buffer for $1 \mathrm{~h}$. Specimens were then dehydrated through a graded series of ethanol solutions and embedded in Agar 100 resin (Agar Aids, Cambridge, UK). Ultrathin sections were stained with uranyl acetate and lead citrate and observed with a Philips 208 electron microscope at $80 \mathrm{kV}$ as described previously. ${ }^{37}$

Acknowledgements. We thank the whole Bioimaging facility of the University of Manchester for the support provided and Martin Lowe and Saska Ivanova for their inputs. RK acknowledges Grant NIH/NHLBI (HL080192) and WM of grants from Ministero della Sanita'. MDE research is supported by BBSRC Grant BB/C508469.

1. Peter ME, Krammer PH. The CD95(APO-1/Fas) DISC and beyond. Cell Death Differ 2003; 10: $26-35$.

2. Chipuk JE, Green DR. Do inducers of apoptosis trigger caspase-independent cell death? Nat Rev Mol Cel Biol 2005; 6: 268-275.

3. Algeciras-Schimnich A, Shen L, Barnhart BC, Murmann AE, Burkhardt JK, Peter ME. Molecular ordering of the initial signaling events of CD95. Mol Cell Biol 2002; 22: 207-220.

4. Lee KH, Feig C, Tchikov V, Schickel R, Hallas C, Schutze S et al. The role of receptor internalization in CD95 signaling. EMBO J 2006; 25: 1009-1023.

5. Cristea IM, Degli Esposti M. Membrane lipids and cell death: an overview. Chem Phys Lipids 2004; 129: 133-160.

6. Levine B, Yuan J. Autophagy in cell death: an innocent convict? J Clin Invest 2005; 115 2679-2688

7. Di Sano F, Ferraro E, Tufi R, Achsel T, Piacentini M, Cecconi F. Endoplasmic reticulum stress induces apoptosis by an apoptosome-dependent but caspase 12-independent mechanism. J Biol Chem 2006; 281: 2693-2700.

8. Hicks SW, Machamer CE. Golgi structure in stress sensing and apoptosis. Biochim Biophys Acta 2005; 1744: 406-414

9. Lowe M, Lane JD, Woodman PG, Allan VJ. Caspase-mediated cleavage of syntaxin 5 and giantin accompanies inhibition of secretory traffic during apoptosis. J Cell Sci 2004; 117 $1139-1150$.

10. Hu W, Xu R, Zhang G, Jin J, Szulc ZM, Bielawski J et al. Golgi fragmentation is associated with ceramide-induced cellular effects. Mol Biol Cell 2005; 16: 1555-1567.

11. Maag RS, Mancini M, Rosen A, Machamer CE. Caspase-resistant golgin-160 disrupts apoptosis induced by secretory pathway stress and ligation of death receptors. Mol Bio Cell 2005; 16: 3019-3027.

12. Garcia-Ruiz C, Colell A, Morales A, Calvo M, Enrich C, Fernandez-Checa JC. Trafficking of ganglioside GD3 to mitochondria by tumor necrosis factor-alpha. J Biol Chem 2002; 277 36443-36448

13. Breckenridge DG, Stojanovic M, Marcellus RC, Shore G. Caspase cleavage product of BAP31 induces mitochondrial fission through endoplasmic reticulum calcium signals, enhancing cytochrome $c$ release to the cytosol. J Cell Biol 2003; 160: 1115-1127.
14. Schneider-Brachert W, Tchikov V, Neumeyer J, Jakob M, Winoto-Morbach S, Held-Feindt $J$ et al. Compartmentalization of TNF receptor 1 signaling: internalized TNF receptosomes as death signaling vesicles. Immunity 2004; 21: 415-428.

15. Sorice M, Circella A, Cristea IM, Garofalo T, Di Renzo L, Alessandri C et al. Cardiolipin and its metabolites move from mitochondria to other cellular membranes during death receptormediated apoptosis. Cell Death Differ 2004: 11: 1133-1145.

16. Sandra F, Degli Esposti M, Magnus M, Knight D, Ndebele K, Khosravi-Far R. TNF-related apoptosis inducing ligand (TRAIL) alters mitochondrial membrane lipids. Cancer Res 2005; 65: $1-12$.

17. Hauri HP, Kappeler F, Andersson H, Appenzeller C. ERGIC-53 and traffic in the secretory pathway. J Cell Sci 2000; 113: 587-596.

18. Marra P, Maffucci T, Daniele T, Tullio GD, Ikehara Y, Chan EK et al. The GM130 and GRASP65 Golgi proteins cycle through and define a subdomain of the intermediate compartment. Nat Cell Biol 2001; 3: 1101-1113.

19. Kenis $\mathrm{H}$, van Genderen $\mathrm{H}$, Bennaghmouch $\mathrm{A}$, Rinia HA, Frederik P, Narula J et al. Cell surface-expressed phosphatidylserine and annexin $\mathrm{A} 5$ open a novel portal of cell entry. J Biol Chem 2004; 279: 52623-52629.

20. Perez-Vilar J, Hidalgo J, Velasco A. Presence of terminal $\mathrm{N}$-acetylgalactosamine residues in subregions of the endoplasmic reticulum is influenced by cell differentiation in culture. J Biol Chem 1991; 266: 23967-23976.

21. Bilyy RO, Stoika RS. Lectinocytochemical detection of apoptotic murine leukemia L1210 cells. Cytometry A 2003; 56: 89-95.

22. Eda S, Yamanaka M, Beppu M. Carbohydrate-mediated phagocytic recognition of early apoptotic cells undergoing transient capping of CD43 glycoprotein. J Biol Chem 2004; 279: 5967-5974.

23. Vetterlein M, Ellinger A, Neumuller J, Pavelka M. Golgi apparatus and TGN during endocytosis. Histochem Cell Biol 2002; 117: 143-150.

24. Kawasaki Y, Saito T, Shirota-Someya Y, Ikegami Y, Komano H, Lee MH et al. Cell deathassociated translocation of plasma membrane components induced by CTL. J Immunol 2000; 164: 4641-4648.

25. Jaiswal JK, Andrews NW, Simon SM. Membrane proximal lysosomes are the major vesicles responsible for calcium-dependent exocytosis in nonsecretory cells. $J$ Cell Biol 2002; 59: 625-635

26. Azuma Y, Taniguchi A, Matsumoto K. Decrease in cell surface sialic acid in etoposide-treated Jurkat cells and the role of cell surface sialidase. Glycocnj J 2000; 17: 301-306.

27. Piller V, Piller F, Fukuda M. Biosynthesis of truncated O-glycans in the T cell line Jurkat. Localization of O-glycan initiation. J Biol Chem 1990; 265: 9264-9271

28. Bell AW, Ward MA, Blackstock WP, Freeman HN, Choudhary JS, Lewis AP et al. Proteomics characterization of abundant Golgi membrane proteins. J Biol Chem 2001; 276: 5152-5165.

29. Schamel WW, Kuppig S, Becker B, Gimborn K, Hauri HP, Reth M. A high-molecularweight complex of membrane proteins BAP29/BAP31 is involved inn the retention of membrane-bound IgD in the endoplasmic reticulum. Proc Natl Acad Sci USA 2003; 100: 9861-9866.

30. Szczesna-Skorupa E, Kemper B. BAP31 is involved in the retention of cytochrome P450 $2 \mathrm{C2}$ in the endoplasmic reticulum. J Biol Chem 2005; 281: 4142-4148.

31. Jones LJ, Upson RH, Haugland RP, Panchuk-Voloshina N, Zhou M, Haugland RP. Quenched BODIPY dye-labeled casein substrates for the assay of protease activity by different fluorescence measurement. Anal Biochem 1997; 251: 144-152.

32. Assfalg-Machleidt I, Rothe G, Klingel S, Banati R, Mangel WF, Valet G et al. Membrane permeable fluorogenic rhodamine substrates for selective determination of cathepsin $\mathrm{L}$. Biol Chem Hoppe Seyler 1992; 373: 433-440.

33. Collette J, Bocock JP, Ahn K, Chapman RL, Godbold G, Yeyeodu S et al. Biosynthesis and alternate targeting of the lysosomal cysteine protease cathepsin L. Int Rev Cytol 2004; 241: $1-51$

34. Michallet MC, Saltel F, Flacher M, Revillard JP, Genestier L. Cathepsin-dependent apoptosis triggered by supraoptimal activation of $\mathrm{T}$ lymphocytes: a possible mechanism of high dose tolerance. J Immunol 2004; 172: 5405-5414.

35. Dadsetan S, Shishkin V, Fomina AF. Intracellular $\mathrm{Ca} 2+$ release triggers translocation of membrane marker $\mathrm{fm} 1-43$ from the extracellular leaflet of plasma membrane into endoplasmic reticulum in T lymphocytes. J Biol Chem 2006; 280: 16377-16382.

36. Falasca L, Bergamini A, Serafino A, Balabaud C, Dini L. Human Kupffer cell recognition and phagocytosis of apoptotic peripheral blood lymphocytes. Exp Cell Res 1996; 224: 152-162.

37. Matarrese P, Tinari A, Mormone E, Bianco GA, Toscano MA, Ascione B et al. Galectin-1 sensitizes resting human $T$ lymphocytes to Fas (CD95)-mediated cell death via mitochondrial hyperpolarization, budding, and fission. J Biol Chem 2005; 280: 6969-6985.

38. Degli Esposti M, Cristea IM, Gaskell SJ, Nakao Y, Dive C. Pro-apoptotic Bid binds to monolysocardiolipin, a new molecular connection between mitochondrial membranes and cell death. Cell Death Differ 2003; 10: 1300-1309. 